\title{
Neumonía adquirida en la comunidad en adultos, en el curso de la campaña de invierno 2003 en el Hospital San Juan de Dios
}

\author{
KAREN DINTRANS A.*, CARMEN LUZ ANDRADE V.***, \\ JUAN EDUARDO SÁNCHEZ V.* y JUAN MENDOZA N.*****
}

\section{COMMUNITY ACQUIRED PNEUMONIA IN ADULTS DURING WINTER 2003 CAMPAIGN AT A SANTIAGO GENERAL HOSPITAL}

Community acquired pneumonia (CAP) continues being a condition of high prevalence, and potentially lethal. Streptococcus pneumoniae is the most frequent etiological agent of CAP. The objective of the present study is to describe the clinical and demographic characteristics, as well as the evolution, of adult patients admitted with CAP to the service of medicine of a Santiago general hospital (Hospital San Juan de Dios). 200 adults admitted with CAP diagnosis during winter 2003, were retrospectively studied. In 170 out of these 200 patients, diagnosis was confirmed. In average the patients were 68 years old. 51\% of patients older than 65 y.o. had received influenza vaccination. High blood pressure, diabetes mellitus and heart failure were the most prevalent non respiratory comorbidities $(59,31$ and 22\% respectively). Chronic obstructive pulmonary disease was the most prevalent respiratory comorbidity (19\%). The antibiotic therapy by election was a third generation cephalosporin, associated with a macrolide. The switch therapy was done in 140 patients (82\%), using an antipneumococcal fluoroquinolone in $90 \%$ of the cases. The median period of hospitalization was 6 days. $40 \%$ of the patients required to be transferred to a clinical unit of higher complexity. In patients having a confirmed diagnosis of community acquired pneumonia lethality rose up to $11 \%$. In only one of the patients, a Streptococcus pneumoniae resistant to Penicillin was isolated in the blood culture. Conclusion: CAP is a prevalent disease, especially in elderly patients, with specific clinical and demographic characteristics. CAP's evolution and mortality rate can be known and modified.

Key words: community pneumonia, bacterial, treatment.

\section{RESUMEN}

La neumonía adquirida en la comunidad (NAC) continúa siendo una condición de alta prevalencia y potencialmente letal. El Streptococcus pneumoniae, es el agente etiológico más frecuente de las NAC. El objetivo del presente estudio, es describir las características clínicas y demográficas, así como también la evolución, de los pacientes adultos ingresados por NAC al servicio de medicina del Hospital San Juan de Dios. Nosotros estudiamos en forma retrospectiva a 200 pacientes adultos ingresados con diagnóstico de NAC. Se confirmó el diagnóstico en 170 de ellos. El promedio de edad fue de 68 años. La vacunación antiinfluenza en mayores de 65 años fue de 51\%. De la comorbilidad no respiratoria, la hipertensión arterial y la diabetes mellitus, fueron las más prevalentes (59\% y 31\% respectivamente),

* Servicio de Medicina, Hospital San Juan de Dios.

** Jefe, Unidad de Enfermedades Respiratorias.

*** Jefe, Servicio de Medicina. 
seguidas por la insuficiencia cardíaca (22\%). La comorbilidad respiratoria más frecuente fue la enfermedad pulmonar obstructiva crónica (EPOC): 19\%. El esquema antibiótico de elección fue una cefalosporina de tercera generación asociada a un macrólido. La terapia secuencial se realizó en 140 pacientes (82\%), empleándose una fluoroquinolona antineumocócica en $90 \%$ de los casos. La mediana de los días de hospitalización fue de 6 días. Un 40\% de los pacientes requirió traslado a una unidad de mayor complejidad. La letalidad asociada a los pacientes con diagnóstico confirmado de NAC, llegó al 11\%. En solo un paciente se aisló un Streptococcus pneumoniae resistente a la penicilina en los hemocultivos. Conclusión: La NAC es una entidad prevalente especialmente en pacientes adultos mayores, con caracteristicas clínicas y demográficas específicas, cuya mortalidad y curso clínico se pueden conocer y modificar.

Palabras claves: neumonía de la comunidad, bacteriana, tratamiento.

\section{INTRODUCCIÓN}

La neumonía adquirida en la comunidad (NAC) continúa siendo una condición de alta prevalencia y potencialmente letal, aún en la era post-antibiótica. Se estima que en Estados Unidos existen 5,6 millones de casos anuales, estando la incidencia alrededor de 12 por 1.000 adultos por año. El número de pacientes que requiere hospitalización por esta causa es de 258 personas por 100.000 habitantes, y 962 por 100.000 personas mayores de 65 años $^{1,2}$. En Chile durante 1999, la neumonía constituyó la tercera causa específica de muerte en ambos sexos, con una tasa de mortalidad de 46,4 por 100.000 habitantes. Ese mismo año nació como un programa del Ministerio de Salud (MINSAL), la "Campaña de Invierno" tanto para niños como para adultos, con el fin de destinar recursos específicos para el manejo de las enfermedades respiratorias durante el periodo de invierno.

De esta forma, la NAC representa un importante problema de salud pública, considerando su elevada prevalencia y letalidad, lo que se traduce en un alto costo económico ${ }^{3}$. En el año 2001 egresaron desde los hospitales del Servicio Nacional de Salud 68.874 pacientes con diagnostico de neumonía, de los cuales 30.723 , correspondían a mayores de 20 años de edad (datos obtenidos del Servicio de Salud Metropolitano Occidente, SSMOc).

La Sociedad Americana del Tórax (ATS), ha elaborado guías para el manejo de los adultos con NAC, clasificándola en cuatro grupos, según presencia de comorbilidad y de factores modificables, necesidad o no de manejo intrahospitalario o requerimiento de Unidad de Cuidados Intensivos. En cada grupo se distinguen los patógenos más probables y el tratamiento antibiótico empírico sugerido ${ }^{2}$. De esta forma, teniendo como base la guía de la ATS de $1993^{4}$, la Sociedad Chilena de Enfermedades Respiratorias publicó sus propias recomendaciones para el tratamiento de la neumonía comunitaria en adultos, no obstante, el criterio clínico debe primar al momento de tomar las decisiones fina$\operatorname{les}^{5,6}$.

La confirmación del patógeno que causa la NAC, sólo se obtiene en menos de la mitad de los casos. El espectro de los gérmenes causantes de NAC no ha variado, y es así, como el Streptococcus pneumoniae, continúa siendo el responsable de la mayoría de los pacientes afectados por esta patología. Se ha determinado que está presente en el 5 al $18 \%$ de los casos y, en un porcentaje aún mayor, cuando se han utilizado técnicas de diagnóstico más agresivas para identificarlo ${ }^{7,8}$.

La mortalidad de los pacientes ambulatorios con NAC es baja (menor a 1-5\%), sin embargo, en aquellos pacientes que requieren hospitalización llega a ser entre un $10-25 \% \mathrm{y}$, se eleva a un $40 \%$ en los que ingresan a una unidad de cuidados intensivos ${ }^{2}$. Las bacterias más frecuentemente asociadas con muerte son el Streptococcus pneumoniae y la Legionella pneumophila. Los factores clínicos predictores de mortalidad definidos por la ATS en el 2001 son edad por sobre los 65 años, coexistencia de enfermedades crónicas, (pulmonar, cardiovascular, renal, cerebro vascular, etc) frecuencia respiratoria mayor o igual a 30 por min, presión diastólica menor o igual a $60 \mathrm{~mm} \mathrm{Hg}$ o sistólica menor o igual a $90 \mathrm{~mm} \mathrm{Hg}$, pulso mayor o igual a 125 por min, temperatura menor de $35^{\circ} \mathrm{C}$ o mayor o igual a $40^{\circ} \mathrm{C}$ y alteración del estado de conciencia. Otros factores que predicen un incremento de la mortalidad son los hallazgos en el laboratorio de neutropenia o neutrofilia, insuficiencia respiratoria, falla renal, hematocrito menor a $30 \%, \mathrm{pH}$ arterial menor de 7,35 y, evidencias radiográficas de compromiso multilobar, 
derrame pleural o rápida progresión de infiltrados radiológicos ${ }^{2,9}$.

En los pacientes de mayor edad, quienes constituyen el grupo de adultos con mayor riesgo de afección por NAC, la infección con virus influenza es un factor crítico, en ellos es apropiado el uso de vacuna antiinfluenza y antineumocócica $^{10}$. Se ha demostrado que la vacunación antiinfluenza está asociada con una reducción de riesgo de hospitalización por enfermedad cardíaca, cerebro vascular, y por neumonía, así como también reduce el riesgo de muerte por estas causas durante la temporada de influen$\mathrm{za}^{11,12}$. Además, la vacuna antineumocócica reduce la frecuencia de infección neumocócica invasiva y de bacteremia ${ }^{13}$. Los datos entregados por el MINSAL, a través del departamento de epidemiología, indican que la cobertura de vacunación antiinfluenza es de un $95 \%$ a partir del año 2001.

Considerando, que la NAC es un importante causa de morbi-mortalidad en los adultos, y que constituye un motivo frecuente de hospitalización y gasto de recursos, se diseñó este análisis descriptivo con el objeto de identificar las características de los pacientes adultos que ingresaron con diagnóstico de NAC al hospital San Juan de Dios durante la campaña de invierno 2003, conocer el manejo y la evolución clínica de ellos. Según estos datos se podrán determinar los recursos necesarios y las metas a seguir para la campaña de invierno 2004.

\section{MATERIAL Y MÉTODOS}

En el periodo comprendido entre el 24 de abril y el 22 de agosto 2003, se evaluaron los pacientes adultos, que ingresaron con diagnóstico de NAC al servicio de medicina del hospital San Juan de Dios. A ellos, se les aplicó el protocolo de "epicrisis de enfermedades respiratorias del adulto campaña de invierno" del departamento de epidemiología del Servicio de Salud Metropolitano Occidente. El protocolo incluye datos como identificación del paciente, fecha de ingreso y egreso, vacunación antiinfluenza, antecedentes de comorbilidad, signos vitales y estado de conciencia al ingreso, exámenes de laboratorio como recuento de leucocitos, función renal, hematocrito, gases en sangre arterial (GSA) u oximetría, hemocultivos, radiografía de tórax, esquema antibiótico inicial y terapia secuencial a oral, lugar de hospitalización (sala común o unidades de mayor complejidad) y condición de egreso. Se excluyeron del estudio los pacientes con diagnóstico VIH positivo o SIDA confirmados por el
Instituto de Salud Pública. El diagnóstico de NAC se confirmó en aquellos casos que cumplían con los criterios diagnósticos descritos en las normas de manejo de NAC del hospital San Juan de Dios 2003, como lo son el inicio de los síntomas $48 \mathrm{~h}$ previo al ingreso, sin antecedentes de hospitalización en las dos semanas previas, presencia de tos con expectoración purulenta, fiebre y dificultad respiratoria. El diagnóstico clínico se confirmó con la radiografía de tórax con la aparición de nuevos infiltrados radiográficos. El registro de los datos y el análisis posterior se realizó en programa Epi Info 2002.

\section{RESULTADOS}

- Características generales de la población: Durante el periodo de estudio se aplicó el protocolo a 200 pacientes hospitalizados en el servicio de medicina con diagnóstico de NAC. Se confirmó el diagnóstico de NAC en 170 de ellos (85\% de los casos), descartándose en 22 (11\% de los casos) y quedando 8 pacientes $(4 \%$ de los casos) en un grupo de NAC probable, ya que en ellos, ni los elementos clínicos ni radiológicos permitieron confirmar el diagnóstico (Figura 1).

Los diagnósticos diferenciales que se prestaron a mayor confusión con neumonía, fueron el edema pulmonar agudo y la descompensación de EPOC. El análisis de los resultados se hizo en los 170 casos en que se confirmó el diagnóstico. Las características demográficas se muestran en la Figura 2. El mayor porcentaje de pacientes provenía de la comuna de Cerro Navia (26\%) seguido por la de Pudahuel (24\%), luego de Quinta Normal (16\%) y posteriormente de Lo Prado (13\%). La vía de ingreso al servicio

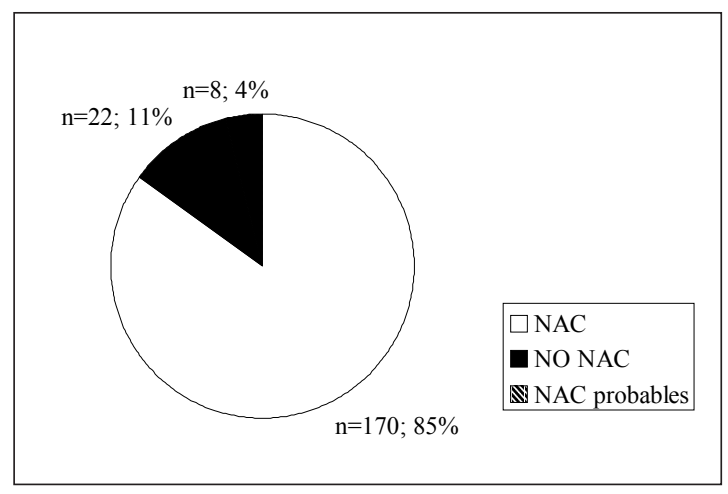

Figura 1. Distribución de pacientes según confirmación de diagnóstico de neumonía adquirida en la comunidad (NAC) al ingreso $(\mathrm{n}=200)$. 


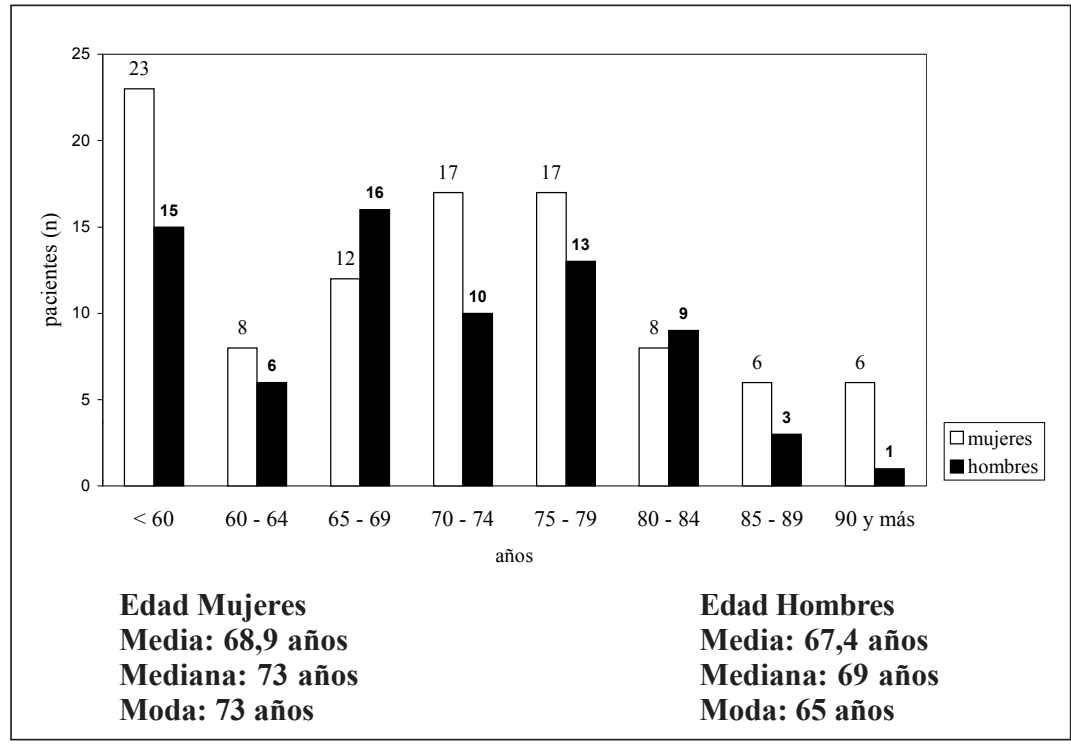

Figura 2. Distribución de pacientes con neumonía adquirida en la comunidad (NAC) confirmada según edad y sexo $(\mathrm{n}=$ 170).

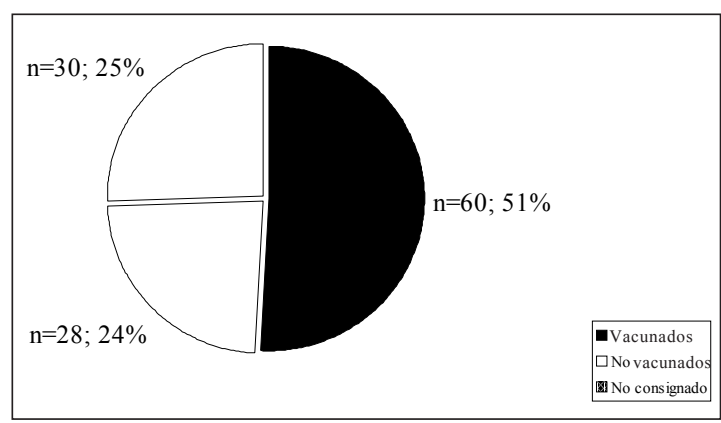

Figura 3. Distribución de pacientes $>65$ años con neumonía adquirida en la comunidad según presencia de vacunación antiinfluenza. Son 118 pacientes los mayores de 65 años que eran la población beneficiaria de la vacuna antiinfluenza. de medicina de gran parte de los pacientes (89\%) fue a través, del servicio de urgencia del hospital y el resto, por medio de los policlínicos de especialidades y otros servicios. La cobertura de la vacunación antiinfluenza 2003 en pacientes con NAC mayores de 65 años se muestra en la Figura 3.

- Evaluación clínica: La comorbilidad estuvo presente en 132 pacientes (Tabla 1). Cabe destacar, que se consideró limitación crónica al flujo aéreo (LCFA) a la obstrucción bronquial debido a daño pulmonar como secuelas de tuberculosis o bronquiectasias. El antecedente de tabaquismo estuvo presente en más de un tercio de los pacientes $(36 \%)$.

Tabla 1. Presencia de comorbilidad en los pacientes con neumonía adquirida en la comunidad

Comorbilidad

Respiratoria

respiratoria
$77,6 \%$ de los pacientes

(\%)

EPOC

LCFA*

Hipertensión arterial $\quad 59$

Diabetes Mellitus 31

Insuficiencia Cardíaca $\quad 22$

Insuficiencia Renal

*LCFA: limitación crónica al flujo aéreo como secuela de tuberculosis o bronquiectasias. 


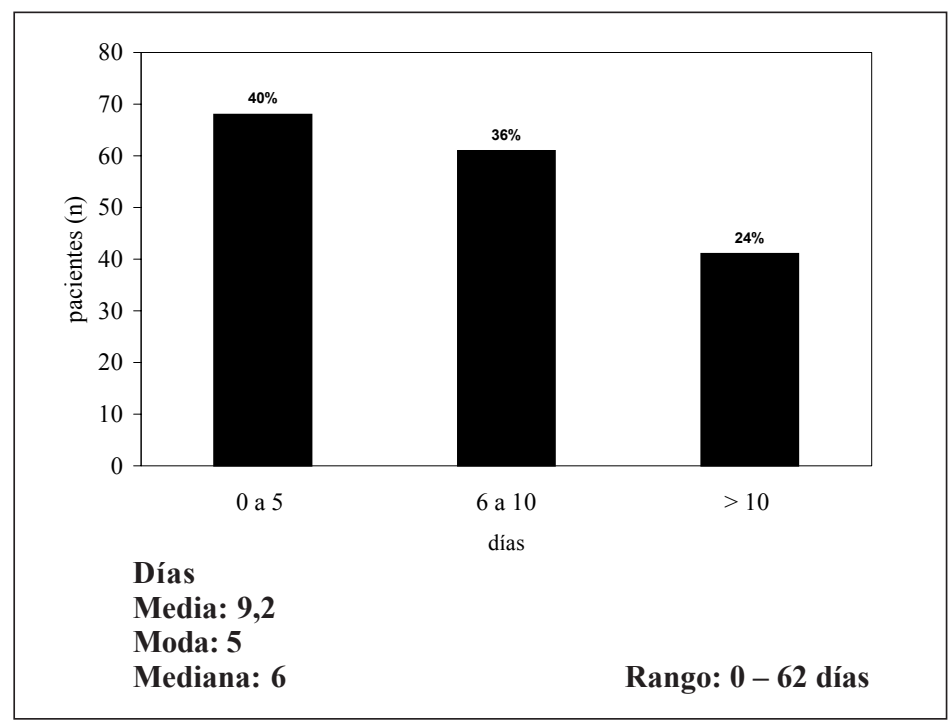

Figura 4. Distribución de pacientes con neumonía adquirida en la comunidad según días de hospitalización en servicio de medicina $(n=170)$. Hubo sólo un paciente fallecido en el primer día de ingreso, el resto falleció después de las $24 \mathrm{~h}$ de hospitalización. Al extraerlo del cálculo de la media, se obtiene el mismo valor.
En el $100 \%$ de los pacientes se realizó radiografía de tórax, describiéndose derrame pleural en 36 de los 170 casos $(21 \%)$.

Los esquemas antibióticos utilizados se enmarcaron dentro de las normas de manejo de NAC del hospital, con un inicio en el $100 \%$ de los casos en forma empírica. El esquema más utilizado fue el de una cefalosporina de tercera generación (ceftriaxona) más un macrólido (eritromicina o claritromicina). Sin embargo, en ocasiones se usó más de un esquema antibiótico durante la estadía hospitalaria. La terapia secuencial a antibiótico oral se logró realizar en 140 pacientes $(82 \%)$, empleándose en más del 90\% de los casos Levofloxacino, en promedio a los 5,6 días (mediana de 5 días). En los 30 restantes, se completó el tratamiento con antibiótico endovenoso, en algunos casos debido a que no se disponía de antibiótico oral para el cambio, no estaba disponible la vía enteral o bien por que las condiciones del paciente no permitían sustituir la terapia intravenosa.

- Lugar de hospitalización y condición de egreso: los días de estada se muestran en la Figura 4. Los motivos que prolongaron la hospitalización fueron principalmente de carácter social. Del total de las NAC derivadas al servicio de medicina, un 36\% requirió traslado a una unidad de cuidados complejos (57 pacientes ingresaron a la unidad de cuidados intermedios, y 3 ingresaron a la unidad de cuidados intensivos, los cuales posteriormente llegaron a las salas de medicina) y un 60\% (103) recibió tratamiento sólo en la sala común. El 4\% restante requirió al

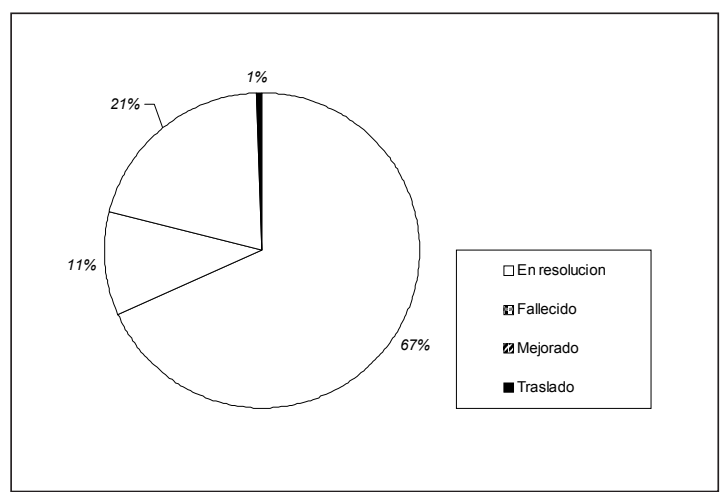

Figura 5. Distribución de pacientes con neumonía adquirida en la comunidad según condición de egreso.

ingreso, manejo en sala crítica del servicio de urgencia. La letalidad asociada a los pacientes que ingresaron por NAC llegó al 11\% (Figura 5).

- Rendimiento diagnóstico de los hemocultivos: Se tomaron dos hemocultivos en 121 pacientes. Sólo se aisló en un paciente un Streptococcus pneumoniae resistente a la penicilina (Tabla 2).

\section{DISCUSIÓN}

Los criterios de hospitalización, se basaron en los factores de gravedad propuestos por la ATS, y que se utilizan en otros hospitales del país ${ }^{2,14}$.

La confirmación del diagnóstico fue retrospectiva, analizando el conjunto de datos con un especialista broncopulmonar, lo que permitió 
Neumonía en adultos. Campaña invierno 2003 - K. Dintrans A. et al.

Tabla 2. Resultados de hemocultivos tomados al ingreso en 121 pacientes, según condición de egreso

\begin{tabular}{lcccc}
\hline Resultados de los hemocultivos & $\begin{array}{c}\text { vivos } \\
\text { (n) }\end{array}$ & $\begin{array}{c}\text { fallecidos } \\
(\mathbf{n})\end{array}$ & $\begin{array}{c}\text { total } \\
(\mathbf{n})\end{array}$ & $\%$ \\
\hline Negativos & 74 & 9 & 83 & 69 \\
Positivos & & & & \\
$\quad$ Contaminados & 26 & 0 & 26 & 21,6 \\
$\quad$ Klebsiella pneumoniae & 0 & 1 & 1 & 0,8 \\
S. pneumoniae Resistente & 1 & 0 & 1 & 0,8 \\
S. pneumoniae Sensible & 5 & 0 & 5 & 4,1 \\
S. alfa hemolitico & 1 & 0 & 1 & 0,8 \\
S. aureus MS & 0 & 1 & 1 & 0,8 \\
$\quad$ Salmonella tiphy & 0 & 1 & 1 & 0,8 \\
Otro (no identificado) & 1 & 0 & 1 & 0,8 \\
Total & 109 & 12 & 121 & 100 \\
\hline
\end{tabular}

excluir un porcentaje no despreciable de pacientes que no correspondían a NAC, lo que se traduce en un mejor rendimiento de los recursos destinados a la campaña de invierno.

Del total de los pacientes hospitalizados durante la campaña de invierno 2003, la gran mayoría corresponden a pacientes adultos mayores, segmento etáreo que está asociado a un incremento en el riesgo de muerte y de un curso complicado de la $\mathrm{NAC}^{1,7}$.

Los datos obtenidos no permiten asegurar una cobertura suficiente de la vacunación en los grupos de riesgo, como lo son los mayores de 65 años. Es preocupante que en el $25 \%$ de los pacientes no se pueda conocer la presencia o ausencia de vacunación antiinfluenza. La dificultad para conseguir el antecedente de vacunación, se debe a varios factores, dentro de los cuales destaca: la falta del registro en la ficha clínica, el desconocimiento del dato por el paciente o por sus familiares o bien, porque el paciente no se encuentra en condiciones de aportar datos. Las razones expresadas por los pacientes que no se vacunaron, incluyen: ignorancia con respecto a la indicación, rechazo individual y contraindicación al momento de la campaña.

La distribución de las comunas en términos numéricos, requiere de un análisis ulterior, ya que, podrían existir diferencias en cuanto al número de habitantes, condición social, edades de la población, existencia de salas de apoyo respiratorio de adultos (SARA) y servicio de atención primaria de urgencia (SAPU), capacitación del personal médico, etc.
En cuanto a los días de estadía por NAC, se encontró una menor estadía que el promedio de días habitual en el servicio de medicina (que está alrededor de 8 días), lo cual es esperable por tratarse de una patología aguda. El objetivo de reducir los días de hospitalización, tal como se describe en algunas publicaciones, implica también reducir los riesgos de infecciones intrahospitalarias $^{15}$.

Respecto al tratamiento antibiótico utilizado, destaca la reducción de los esquemas a prácticamente uno, como es la asociación de una cefalosporina de tercera generación (ceftriaxona) y un macrólido, de manera similar a lo sugerido por la ATS y la Sociedad Americana de Enfermedades Infecciosas (IDSA) ${ }^{2,16}$. Reconociendo el alto costo de las terapias antibióticas endovenosas y la prolongación de los días de hospitalización, se ha adoptado, al igual que en centros extranjeros, la modalidad de terapia endovenosa abreviada si la respuesta clínica es favorable, seguida por un antibiótico vía oral con iguales características y espectro antimicrobiano ${ }^{15}$. Para tener éxito en la terapia, todos los pacientes que se hospitalicen debieran recibir la primera dosis de antibióticos antes de las 8 horas de ingresados al hospital e idealmente de inmediato una vez que se plantea el diagnóstico ${ }^{2}$.

Llama la atención la gran cantidad de pacientes con enfermedades crónicas, lo que podría estar en relación a la edad de la población estudiada, que en su mayoría corresponden a pacientes adultos mayores. El hábito de fumar estuvo presente en más de un tercio de los 
pacientes, dato que causa preocupación, ya que el tabaquismo se ha descrito como el factor de riesgo independiente más importante para una infección pneumocócica invasiva en los adultos inmunocompetentes no ancianos ${ }^{17}$. La condición de tener comorbilidad no permitió establecer un pronóstico de gravedad. Debido a esto, se estableció la aplicación en forma retrospectiva de un puntaje de gravedad, como lo es el índice de Fine $^{18}$, para su validación, y posterior empleo en la campaña de invierno 2004.

La radiografía de tórax es una herramienta diagnóstica fundamental para la NAC. Sin embargo, es un procedimiento con baja especificidad para orientar hacia un determinado agente etiológico y requiere de un diagnóstico diferencial acucioso con el compromiso pulmonar de origen cardiovascular y otros como la fibrosis pulmonar. En el estudio se observó que la toma de la radiografía de tórax llegó al 100\% de los pacientes ingresados, siendo interpretada por un médico internista. El derrame pleural, se evidenció en un porcentaje menor ( $21 \%$ de los casos) al comunicado en otras series donde alcanza entre un 30 y $50 \%$ de las $\mathrm{NAC}^{1,7}$.

La letalidad asociada a NAC recogida por este estudio de un $11 \%$, fue menor a la del año anterior, ya que éste valor alcanzó al $16 \%$ en el servicio de medicina y al $32 \%$ en el servicio de urgencia del hospital en el año 2002. Estos valores están dentro de lo esperado para esta patología, según se informa en publicaciones nacionales e internacionales ${ }^{13,19,20}$. Es necesario destacar que el $89 \%$ de los casos fallecieron en el servicio de tratamiento intensivo, lo cual revela la mayor gravedad de los pacientes. En dos de ellos la causa de la muerte no fue atribuida directamente a la NAC (accidente vascular encefálico hemorrágico y tromboembolismo pulmonar masivo).

La positividad de los hemocultivos en la literatura es aproximada al 11\%, y de ellos un $67 \%$ corresponden a Streptococcus pneumoniae, lo que ha significado que la relación costo/beneficio de este examen bacteriológico, sea ampliamente cuestionada. La toma rutinaria de hemocultivos, raramente contribuye en forma significativa al manejo clínico de la NAC. En estudios recientes, no se han comunicado diferencias en cuanto a la letalidad en los pacientes con hemocultivos positivos o negativos y en aquellos con Streptococcus pneumoniae resistentes o sensible a antibiótico ${ }^{1,13,21-23}$. A la luz de los resultados obtenidos en el transcurso de la campaña, con un alto porcentaje de hemocultivos negativos y otros tantos contaminados, se determinó dejar de tomar hemocultivos como norma a los pacientes que ingresaran con diagnóstico de NAC, lo que redujo la información de estos cultivos a 121 pacientes.

El valor diagnóstico del test de Gram y los cultivos de expectoración se ha discutido por más de dos décadas. Se ha comunicado que los cultivos de esputo resultan ser negativos entre un 30 a $65 \%$, lo que podría explicarse en algunos casos por la presencia de tos no productiva o por uso de antibióticos previo a la hospitalización. Tal vez, la única ventaja de hacer el Gram, sea la de ajustar la terapia antibiótica de inicio, cuando este test muestra un microorganismo que no estaría cubierto por el esquema antibiótico empírico usualmente utilizado, y la del cultivo, sea la de detectar los Streptococcus pneumoniae resistente a la penicilina ${ }^{1,2}$. En Chile, el porcentaje de resistencia global de $S$. pneumoniae en pacientes adultos varía entre 3,0 y $8,4 \%$ dependiendo del grupo etáreo. No se han descrito cepas resistentes a cefalosporinas ${ }^{24}$. La cobertura de gérmenes anaerobios está en discusión, y no se recomienda de rutina, salvo en aquellos pacientes con sepsis oral.

Deben destacarse adelantos recientes en el estudio etiológico de las NAC como la detección de antígenos capsulares de Streptococcus pneumoniae en expectoración o líquido pleural (técnica de aglutinación por látex), la introducción de estudios serológicos para el diagnóstico de agentes atípicos, la inclusión de técnicas rápidas para detección viral en muestras de expectoración y la capacidad de reconocer agentes mediante antígenos específicos en orina. El alto costo de estos estudios, limitan su uso a casos complicados o severos ${ }^{7}$.

\section{CONCLUSIÓN}

La NAC es una entidad prevalente especialmente en pacientes adultos mayores, con características clínicas y epidemiológicas específicas, cuya mortalidad y curso clínico se pueden conocer e intervenir. Los resultados de este estudio, permiten orientar hacia las metas propuestas para la campaña de invierno 2004, en términos de la importancia de contar con registros fidedignos que permitan tomar decisiones fundamentadas, comparar año a año los datos encontrados e identificar tendencias para el año siguiente, establecer normas de manejo, y la aplicación de protocolos de ingreso y de tratamiento único para los pacientes, con el fin de reducir la mortalidad por NAC. 


\section{BIBLIOGRAFÍA}

1.- BARTLETT J G, MUNDY L M. Community-Adquired. N Engl J Med 1995; 333: 1618-24.

2.- Guidelines for the Management of Adults with Community-acquired Pneumonia. Am J Respir Crit Care Med 2001; 163: 1730-54.

3.- SALDÍAS F, MARDÓNEZ J M, MARCHESSE M, VIVIANI P, FARÍAS G, DÍAZ A. Neumonía adquirida en la comunidad en el adulto hospitalizado. Cuadro clínico y factores pronósticos. Rev Méd Chile 2002; 130: 1373-82.

4.- NIEDERMAN M S, BASS J B JR, CAMPBELL G D, FEIN A, GROSSMAN R, MANDELL L et al. Guidelines for the initial management of adults with community acquired pneumonia: diagnosis, assessment of severity, and initial antimicrobial therapy. American Thoracic Society. Medical Section of the American Lung Association. Am Rev Respir Dis 1993; 148: 1418-26.

5.- Sociedad Chilena de Enfermedades Respiratorias. Consenso Nacional en Neumonías del Adulto Adquiridas en la Comunidad. Rev Chil Enf Respir 1999; 15: 70105.

6.- NARETTO E, SOTO M, GUTIÉRREZ M, CARRASCO C. Estrategias terapéuticas en las neumonías adquiridas en la comunidad. Bol Hosp San Juan de Dios 1999; 46: $13-8$

7.- FICA A. Enfoque diagnóstico de las neumonías adquiridas en la comunidad en pacientes adultos. Rev Chil Infect 2002; 19: 156-66.

8.- BROWN P D. Community-acquired pneumonia. Lancet 1998; 352: 1295-302.

9.- LIM W S, VAN DER EERDEN M M, LAING R, BOERSMA W G, KARALUS N, TOWN G I et al. Defining community acquired pneumonia severity on presentation to hospital: an international derivation and validation study. Thorax 2003; 58: 377-82.

10.- JANSSENS J P, KRAUSE K H. Pneumonia in the very old. Lancet Infect Dis 2004; 4: 112-24.

11.- NICHOL K, NORDIN J, MULLOOLY J, LASK R, FILLBRANDT K, IWANE M. Influenza vaccination and reduction in hospitalizations for cardiac disease and stroke among the elderly. N Engl J Med 2003; 348: 1322-32.

12.- JACKSON L, YU O, HECKBERT S, PSATY B, MALAIS D, BARLOW W et al. Influenza vaccination is not associated with a reduction in the risk of recurrent coronary events. Am J Epidemiol 2002; 156: 634-40.

13.- DÍAZ A, TORRES C, FLORES L J, GARCÍA P,
SALDÍAS F. Neumonía neumocócica adquirida en la comunidad en adultos hospitalizados. Rev Méd Chile 2003; 131: 505-14.

14.- JADUE C, FERRARI F. Neumonía adquirida en la comunidad: criterios de hospitalización. Bol Hosp Viña del Mar 1996; 52: 16-20.

15.- FERNÁNDEZ P, SAN MARTÍN L. Neumonía adquirida en la comunidad: Terapia secuencial de cefalosporina intravenosa a cefalosporina oral. Rev Méd Chile 2000; 128: 267-72.

16.- MANDELL L A, BARTLETT J G, DOWELL S F, FILE T M, MUSHER D M, WHITNEY C. Update of Practice Guidelines for the Management of Community-Acquired Pneumonia in Immunocompetent Adults. Clinical Infectious Diseases 2003; 37: $1405-33$

17.- FILE T M. Community-acquired pneumonia. Lancet 2003; 362: 1991-2001.

18.- FINE M J, AUBLE T E, YEALY D M, HANUSA B H, WEISSFELD L A, SINGER D E et al. A prediction rule to identify low-risk patients with communityacquired pneumonia. N Eng J Med 1997; 336: 24350.

19.- MARRAS T K, GUTIÉRREZ C, CHAN C K. Applying a prediction rule to identify low-risk patients with community-acquired pneumonia. Chest 2000; 118: 1339-43.

20.- ZALACAIN R, TORRES A, CELIS R, BLANQUER J, ASPA J, ESTEBAN L et al. Community-acquired pneumonia in the elderly: Spanish multicenter study. Eur Respir J 2003; 21: 294-302.

21.- WILKINSON M, WOODHEAD M A. Guidelines for community-acquired pneumonia in the ICU. Curr Opin Crit Care 2004; 10: 59-64.

22.- DÍAZ A, CALVO M, O'BRIEN A, FARÍAS G, MARDÓNEZ J M, SALDÍAS F. Utilidad clínica de los hemocultivos en pacientes hospitalizados por neumonía adquirida en la comunidad. Rev Méd Chile 2002; 130: 993-1000.

23.- CAMPBELL S, THOMAS M, ANSTEY R, DICKINSON G, ACKROYD-STOLARZ S. The contribution of blood cultures to the clinical management of adult patients admitted to the hospital with communityacquired pneumonia. Chest 2003; 123: 1142-50.

24.- SOLER T, SALAMANCA L, ARBO G, MOLINA E. Estudios de sensibilidad in vitro de cepas de Streptococcus pneumoniae a penicilina y su asociación con factores clínicos y epidemiológicos. Rev Méd Chile 2002; 130: 26-34. 